\title{
Mindfulness and Acceptance in Developmental Disabilities: Introduction to the Special Issue
}

\author{
Richard P. Hastings $\cdot$ Ramasamy Manikam
}

Published online: 7 April 2013

(C) Springer Science+Business Media New York 2013

Mindfulness and acceptance training programs and interventions have rapidly gained widespread international interest, but their use in the field of developmental disabilities has been much slower. However, the field appears to have overcome the inertia associated with new approaches and is on the tipping point of having a major impact. A major reluctance to adopt these new "therapies" may be attributable to the plethora of fads espoused by some professionals in developmental disabilities. Furthermore, there was some question whether individuals with developmental disabilities would have the cognitive capacity to understand and practice some of the foundational concepts associated with mindfulness-based procedures. As attested by the current research, there is now optimism that mindfulness and acceptance procedures may prove to be valuable in assisting parents and paid carers to enhance the quality of life of individuals with developmental disabilities (Noone 2013; Russell 2011). Indeed, there is also limited research that indicates individuals who function at mild, and perhaps moderate, levels of intellectual disabilities may benefit from learning mindfulness-based procedures (Robertson 2011).

The aim of this special issue was to bring together some of the innovative work being done on mindfulness and acceptance training and interventions in the field of developmental disabilities. The first four papers deal with parents and paid carers, followed by three papers that deal with individuals with intellectual disabilities, and one involving both the individuals with intellectual disabilities and their carers. The final paper adds to the rapidly growing number of reviews of the extant mindfulness literature in developmental disabilities (e.g., Harper et al. 2013; Hwang and Kearney 2013a, 2013b).

R. P. Hastings $(\square)$

School of Psychology, Bangor University,

Bangor LL57 2AS Gwynedd, Wales, UK

e-mail: r.hastings@bangor.ac.uk

R. Manikam

American Health and Wellness Institute, Baltimore, MD, USA

\section{Parents and Paid Carers}

There are four papers in this section. Ferraioli and Harris (2013) compared the effects of mindfulness and skills-based parent training programs for parents of children with autism. While both mindfulness-based and behavioral skills approach to parent training have been effective in decreasing parental stress and increasing meaningful parent-child interactions, Ferraioli and Harris provide the first head-to-head comparison in a small pilot study of 15 parents of children with autism who were randomized into two treatment groups. Both groups participated in an 8-week training program that included didactics, discussion, role plays, and homework. Outcome measures of parental stress and global heath were assessed at pretest, posttreatment, and 3-month follow-up. Results showed the mindfulness group alone had a statistically significant improvement on both outcome measures following the 8week course. Positive changes were also noted for the behavioral skills group, but not at a statistically significant level.

Beer et al. (2013) present a correlational study that investigated factors related to parental well-being that could be amenable to intervention. The study was based on a sample of 28 parents of children with autism spectrum disorder. They found that higher levels of mindful parenting were related to lower levels of depressive symptoms and stress, but unrelated to anxiety. The also found that mindful parenting did not mediate the relationship between child behavior problems and parental distress. Overall, the authors suggested that mindful parenting may have benefits for parent's psychological health. What would be interesting is to provide the same parents with an 8-week mindfulness parenting course, as in the Ferraioli and Harris study, and then assess the same correlations. We suspect the correlations would be somewhat different because of better understanding of mindfulness practices by the parents.

Bethay et al. (2013) present a pilot study that evaluated the effects of a combined acceptance and commitment training (ACT) with training in applied behavior analysis (ABA) 
versus training in $\mathrm{ABA}$ alone on 34 staff that worked with individuals with intellectual disabilities. The combined training included $6 \mathrm{~h}$ of $\mathrm{ACT}$ and $3 \mathrm{~h}$ of ABA, and the control condition included $9 \mathrm{~h}$ of ABA training. Each of the conditions consisted of three 3-h group-training sessions, provided at weekly intervals for 3 weeks. Outcome measures included general health and occupational stress. Results showed that only those staff that reported practicing the ACT techniques consistently exhibited decreased general distress relative to controls at posttest, but not at a 3-month follow-up. Those who participated in the combined ACT and ABA training reported significantly larger reductions in distress than those who participated in the ABA training alone. However, there was no statistical difference in the reported frequency of thoughts and feelings indicative of burnout across the two groups. Bethay et al. (2013) suggested that their findings support previous research on ACT interventions for staff who work with individuals with intellectual disabilities.

In the final paper in this section, Brooker et al. (2013) evaluated the effects of a group-based occupational mindfulness (OM) program on staff who work with people with disabilities. Using a single intervention in a pretest/posttest design, Brooker at al. provided the OM training to 34 staff and assessed the impact of training on a large range of outcomes, including mindfulness, perceived stress, depression, positive and negative affect, global life satisfaction, staff burnout, job satisfaction, perceived quality of life, and compassion and self-compassion. Significant changes due to the OM training were reported in terms of increased positive affect and mindfulness facet of observing and a decrease in extrinsic job satisfaction. However, there was a significant decrease in extrinsic job satisfaction and a significant decrease in negative affect, perceived stress, anxiety, and negative emotional symptoms. There were no significant changes in the other outcome measures. Despite the mixed findings from a relatively small study with a large range of outcome measures, the authors noted that the $\mathrm{OM}$ training produced a range of benefits for the participants.

\section{Individuals with Intellectual Disabilities}

There are three papers in this section. Miodrag et al. (2013) report a unique study on mindfulness intervention with 24 adults with Williams syndrome (WS). As the authors note, mindfulness provides a natural treatment for this population because people with this neurodevelopmental disorder often have high rates of anxiety, and attentional and social disabilities. The mindfulness training was based on the standard mindfulness-based stress reduction (MBSR; KabatZinn 1994). The 24 participants were provided training in three groups, with each group led by a trained mindfulness instructor experienced in working with people with disabilities. The training sessions were of 20-min duration, delivered daily, for five consecutive days. Effectiveness of the training was assessed in terms of physiological, psychological, and behavioral outcomes. Results showed decreased cortisol and self-rated anxiety levels. This was a small pilot study that used a modified version of MBSR, but still showed its utility in reducing stress-related symptoms in adults with WS.

Singh et al. (2013) evaluated the effectiveness of a mindfulness-based smoking cessation program with three adults with mild intellectual disabilities. These three individuals had tried but had failed to quit smoking with other interventions. The mindfulness-based smoking cessation program used in this study included basic concentration meditation, daily intention, mindful observation of thoughts, and Meditation on the Soles of the Feet (Singh et al. 2011). The three individuals self-determined the rate at which they decreased and eliminated their smoking behavior via a changing criterion design. Results showed that all three men were able to gradually eliminate smoking and maintain this result for a year, with follow-up showing that they were able to abstain from smoking for a further 3 years. The authors suggested that the mindfulness-based smoking cessation program may be generally effective in this population and should be evaluated in a randomized controlled trial.

In the third paper in this series, Singh et al. (2013) evaluated the effectiveness of a mindfulness-based procedure, Meditation on the Soles of the Feet, to control both physical and verbal aggression. This procedure is a variation on the single-pointed meditation in which the object of the meditation is the soles of one's feet as opposed to the breath. This was a two-group randomized controlled trial (RCT), with each group undergoing 12-week baseline treatment and one or two follow-up phases. A total of 34 adults were randomly assigned either to the experimental (Meditation on the Soles of the Feet) or a waiting list control condition. At the end of the trial, there was a statistically significant reduction in physical and verbal aggression commensurate with mindfulness-based training, when compared to the waiting list control condition. However, similar but slightly smaller statistically significant reductions in physical and verbal aggression were evident when the same training was introduced in the control condition. This study is the culmination of a series of studies using this procedure that began with an uncontrolled case study, a multiple baseline design proof of concept study, replications of the multiple design study across populations, and finally this RCT. What is impressive is that this group of researchers developed a new mindfulness intervention and systematically built the evidence-base stepwise before attempting a RCT. This kind of patient development and testing of a new intervention would certainly eliminate some of the fads experienced in the field of developmental disabilities. 


\section{Participants' Perspectives on Mindfulness Intervention}

There is little research that has explored the use of mindfulness groups for individuals with intellectual disabilities and their carers. Chapman and Mitchell (2013) qualitatively assessed the views of adults with intellectual disabilities and their carers who attended one of the workshops they presented on Introduction to Mindfulness. The authors conducted a mindfulness program with adults with developmental disability and the staff who care for them. The researchers offered introductory mindfulness workshops for adults with DD, in a community setting, and then used questionnaire and interview methods to gain the adults' perspectives on mindfulness, why they have attended the workshops, and whether they believed mindfulness might help them in their lives. Although it was clear that some of the language used in mindfulness was a challenge, the direct experience of a mindfulness meditation seemed to be a positive way for adults with DD to gain an understanding of the approach. Results suggested that the participants thought that mindfulness may be effective in relieving some of the stress in their daily lives. It is interesting that the participants were positive even with a single 20-min body scan session suggesting that evaluation with a more robust course on mindfulness is warranted.

\section{Review}

The final paper in this special issue reviews some of the extant work that has been published in the last decade on applications of mindfulness in developmental disabilities. Chapman et al. (2013) reviewed 11 studies based on a systematic process and their selection criteria. Their overall assessment was that the extant literature shows promise, but further research is warranted. This review utilized the Evaluative Method for Determining Evidence Based Practice of Reichow et al. (2008) as the basis for evaluating the 11 studies. What is interesting is that this methodology rates single-subject experimental studies as weak in rigor while the same studies would be rated as meeting all the criteria of robust experimental design by single-subject methodologists (Barlow et al. 2009).

In summary, these nine papers provide a sampling of the breadth of research on mindfulness and acceptance that is being done around the world. The findings reported in these studies suggest that further work is definitely warranted given the promising results from the diverse applications of these interventions. Research on a continuum of case studies, single-subject experimental studies, and RCTs are warranted given the recent emergence of this field of research, especially in this population. As research psychologists, we also advocate research on the general mechanisms that may account for the effectiveness of these approaches in this population. However, we will understand if researchers may be hesitant to undertake such research because of the lack of a solid foundation of basic mindfulness research with people with developmental disabilities. In any case, we hope that this special issue provokes discussion, triggers ideas, and leads to the next level of research development.

\section{References}

Barlow, D. H., Nock, M. K., \& Hersen, M. (2009). Single case experimental designs: strategies for studying behavior change. Boston: Allyn and Bacon.

Beer, M., Ward, L., \& Moar, K. (2013). The relationship between mindful parenting and distress in parents of children with as autism spectrum disorder. Mindfulness. doi:10.1007/s12671-012-0192-4.

Bethay, J.S., Wilson, K.G., Schnetzer, L.W., Nassar, \& Bordieri, M.J. (2013). A controlled pilot evaluation of acceptance and commitment training for intellectual disability staff. Mindfulness. doi:10.1007/s12671-012-0103-8.

Brooker, J., Julian, J., Webber, L., Chan, J., Shawyer, F., \& Meadows, G. (2013). Evaluation of an occupational mindfulness program for staff employed in the disability sector in Australia. Mindfulness. doi:10.1007/s12671-012-0112-7.

Chapman, M.J., Hare, D.J., Caton, S., Donalds, D., McInnis, E., \& Mitchell, D. (2013). The use of mindfulness with people with intellectual disabilities: a systematic review and narrative analysis. Mindfulness. doi:10.1007/s12671-013-0197-7.

Chapman, M.J. \& Mitchell, D. (2013). Mindfully valuing people now: an evaluation of Introduction to mindfulness workshops for people with intellectual disabilities. Mindfulness. doi:10.1007/s12671-012-0183-5.

Ferraioli, S.J. \& Harris, S.L. (2013). Comparative effects of mindfulness and skills-based parent training programs for parents of children with autism: feasibility and preliminary outcome data. Mindfulness. doi:10.1007/s12671-012-0099-0.

Harper, S.K., Webb, T.L., \& Rayner, K. (2013). The effectiveness of mindfulness-based interventions for people with intellectual disabilities: A narrative review. Behavior Modification. doi:10.1177/ 014544551347608.

Hwang, Y.-S., \& Kearney, P. (2013a). A systematic review of mindfulness intervention for individuals with developmental disabilities: long-term practice and long lasting effects. Research in Developmental Disabilities, 34, 314-326.

Hwang, Y-S., \& Kearney, P. (2013b). Mindful and mutual care for individuals with developmental disabilities: a systematic literature review. Journal of Child and Family Studies. doi:10.1007/ s10826-012-9707-9.

Kabat-Zinn, J. (1994). Wherever you go, there you are: mindfulness meditation in everyday life. New York: Hyperion.

Miodrag, N., Lense, M.D., \& Dykens, E.M. (2013). A pilot study of a mindfulness intervention for individuals with Williams Syndrome: Physiological outcomes. Mindfulness. doi:10.1007/ s12672-012-0178-2.

Noone, S. J. (2013). Supporting care staff using mindfulness- and acceptance-based approaches. In J. L. Taylor, W. R. Lindsay, R. Hastings, \& C. Hatton (Eds.), Psychological therapies for adults with intellectual disabilities (pp. 207-221). Chichester: Wiley.

Reichow, B., Volkmar, F., \& Cicchetti, D. (2008). Development of the evaluative method for evaluating and determining evidence-based practices in autism. Journal of Autism and Developmental Disorders, 38(7), 1311-1319. 
Robertson, B. (2011). The adaptation and application of mindfulness-based psychotherapeutic practices for individuals with intellectual disabilities. Advances in Mental Health and Intellectual Disabilities, 5(5), 46-52.

Russell, J. (2011). Mindfulness: a tool for parents and children with Asperger's syndrome. Mindfulness, 2, 212-215.

Singh, N.N., Lancioni, G.E., Winton, A.S.W., Karazsia, B.T., Singh, A.D.A., Singh, A.N.A., \& Singh, J. (2013). A mindfulness-based smoking cessation program for individuals with mild intellectual disability. Mindfulness. doi:10.1007/s12671-012-0148-8.
Singh, N. N., Singh, J., Singh, A. D. A., Singh, A. N. A., \& Winton, A. S. W. (2011). Meditation on the soles of the feet for anger management: a trainer's manual. Raleigh: Fernleaf. www.fernleafpub.com.

Singh, N.N., Lancioni, G.E., Karazsia, B.T., Winton, A.S.W., Myers, R.E., Singh, A.N.A., Singh, A.D.A., \& Singh, J. (2013). Mindfulnessbased treatment of aggression in individuals with intellectual disabilities: a waiting list control study. Mindfulness. doi:10.1007/s12671-012-0180-8. 